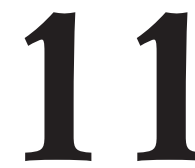

\title{
LA FORMACIÓN DEL PROFESORADO UNIVERSITARIO PARA LA IMPLEMENTACIÓN DE LA EVALUACIÓN POR COMPETENCIAS
}

\author{
(UNIVERSITY'S TEACHERS TRAINING TOWARDS ASSESSMENT BY \\ COMPETENCES)
}

Georgeta Ion

Universidad Autónoma de Barcelona

Elena Cano

Universidad de Barcelona

\section{RESUMEN}

La evaluación centrada en competencias es uno de los retos que la Declaración de Bolonia supone para el profesorado universitario español. Este cambio en las metodologías docentes requiere por parte del profesorado una actividad de formación permanente. El objetivo del estudio que presentamos es analizar en qué medida los programas de formación propuestos por las universidades responden a las necesidades actuales del profesorado universitario, con especial énfasis en la formación para la evaluación por competencias.

Se han realizado entrevistas con responsables de los institutos de formación de cinco universidades españolas. Los resultados indican que, aunque la oferta formativa es amplia, todavía hay pocas experiencias formativas orientadas hacia la evaluación por competencias. Aunque el interés hacia la evaluación es cada vez mayor, todavía predomina la demanda sobre estrategias formativas y los temas «clásicos». Las conclusiones apuntan hacia la necesidad de más esfuerzos para ofrecer una formación coherente y adecuada a los nuevos retos del EEES.

\section{ABSTRACT}

The competency based assessment is one of the challenges academics have to face in the context. This change of paradigm in the didactic strategies implies new teacher training programs. 
The present article aim to analyze how the teachers training programs promoted by the universities respond to the academics needs, especially their need to implement a competency based assessment with the students.

The methods consist in an implementation of in depth interviews with the heads of teachers training institutions from five Spanish universities.

The results pointed out that the teachers training offer related with the assessment strategies based on competencies is still poor. Despite the elevated academics interest in this field, the training methods are still traditional and the topics "classics». The conclusions pointed to the need of more efforts in order to align the teachers training programs to the new Higher Education Area requirements.

\section{INTRODUCCIÓN}

\subsection{El marco del Espacio Europeo de Educación Superior}

La creación del Espacio Europeo de Educación Superior (EEES) (Ministros Europeos de Educación, 1999) ha significado un cambio de paradigma, que implica no sólo transformaciones estructurales del sistema universitario sino también en la dinámica organizativa institucional y en el modo de concebir los procesos de aprendizaje. El sistema de evaluación por competencias es un principio rector de la reforma curricular (EUA, 2010) cuya asunción parece que llevará tiempo. Y es que la universidad española, como ha indicado la propia ministra Garmendia (2009), arrastra déficits muy importantes, entre los que destaca la ausencia de metodologías centradas en el aprendizaje de los alumnos.

Se pretende, entre otras cosas, aumentar la credibilidad social de la universidad, respondiendo a la exigencia de población cualificada. Para ello se propone la adaptación de un sistema de estudios en dos ciclos y el uso de un modo de trabajo basado en el Sistema de Transferencia de Créditos Europeos (ECTS) que supone hacer pivotar el aprendizaje no sobre los contenidos sino sobre las competencias y situar el protagonismo -y la responsabilidad- del aprendizaje, en el alumnado. Este proceso no supone solo un cambio en el estilo de aprendizaje del alumnado sino que requiere por parte del profesorado un cambio de cultura, pasando de un rol de instructor a un rol de facilitador del aprendizaje. La transformación en las tareas de los docentes se hace patente tanto a nivel organizativo como a nivel curricular: desarrollo de metodologías activas e implantación de sistemas de evaluación de los aprendizajes y las competencias (EUA, 2005). 
Cada grupo de profesores que conforma el equipo docente de una titulación debe plantearse cuál/es de las competencias de dicha titulación puede desarrollar desde sus respectivas áreas y cómo va a hacerlo. Pero ello no puede hacerse si no va acompañado de cambios, entre otros ámbitos, en los modos de trabajar y en los horarios, en los espacios y en las estructuras (Valcárcel, 2004).

\subsection{Competencias y EEES}

Entendemos, siguiendo a Perrenoud (2004) que la competencia es la selección y combinación pertinente de conocimientos, habilidades, destrezas, actitudes, valores y normas que permiten dar respuesta a una situación compleja en un contexto determinado.

Estamos de acuerdo con Rué (2007) cuando define la competencia como la capacidad de responder con éxito a las exigencias personales y sociales que nos plantea una actividad o una tarea cualquiera en el contexto del ejercicio profesional. Comporta dimensiones tanto de tipo cognitivo como no cognitivo.

Para aproximarnos a la comprensión del concepto, Cano $(2008,3)$ rescata tres elementos que caracterizan las competencias:

Articulan conocimiento conceptual, procedimental y actitudinal. El ser competente supone, de todo el acervo de conocimiento que uno posee (o al que puede acceder), seleccionar el que resulta pertinente en aquel momento y situación para poder resolver el problema o reto que enfrentamos.

Se desarrollan. Se puede progresar en las competencias con formación inicial, con formación permanente y con la experiencia a lo largo de la vida.

Toman sentido en la acción. La competencia implica la capacidad de resolución en contextos (conocidos o desconocidos), dando respuesta y sentido a situaciones diferentes a las vividas (por lo que no es suficiente la repetición mecánica de "problemas tipo»).

Con este nuevo escenario las universidades se ven obligadas a revisar y actualizar sus programas de estudio y los títulos que ofrecen, adecuándolos a las necesidades de la sociedad y del mercado laboral actual y a las demandas que éste pone frente a los que se incorporan a él, procurando aprendizajes competenciales que lleven a comprender y habitar el mundo y a desarrollar una profesión de modo eficiente. Estos aprendizajes competenciales, auténticos, situados, necesitan de una evaluación acorde a 
los mismos. En la literatura especializada empiezan a aparecer, cada vez, más referencias sobre la evaluación por competencias (Mateo, 2000; Suárez, s/f; De Miguel, 2004; Tejada, 2005; Rama, 2006; Alonso, 2010; Cano, 2011; entre otros) y sobre las implicaciones organizativas que las metodologías de enseñanza-aprendizaje y evaluación tienen sobre los diferentes ámbitos de la vida universitaria (Villardón, 2006; San Pedro, 2009; Gairín, 2009; Olmos \& Rodríguez, 2010; entre otros). Estos estudios coinciden en la necesidad de un cambio cultural del profesorado respecto de los procesos de evaluación y de una mayor formación sobre el tema.

\subsection{La formación como elemento clave para el cambio hacia el EEES y los diseños por competencias}

La formación del profesorado sigue siendo un tema de actualidad desde los diferentes ámbitos de la vida universitaria. Frente a los nuevos retos del EEES, una puesta al día sobre las nuevas demandas docentes, con énfasis en la participación y la motivación hacia la enseñanza, aparecen como elementos imprescindibles para una educación de calidad (García Llamas, 1998).

Los estudios realizados a nivel internacional y español ponen de manifiesto que la formación del profesorado universitario incluye programas que fomentan, por un lado, procesos de desarrollo profesional (Schön, 1987) y, por el otro, el cambio de las concepciones sobre la enseñanza- aprendizaje y evaluación del profesorado (Edwards, Donderis, \& Ballester, 2005). Muchos programas de formación están orientados hacia el desarrollo de competencias del profesorado sobre todo lo que tenga que ver con su actividad en las aulas (Javaloyes, 2009; Espinosa , Jiménez, Olabe \& Basogain, 2010).

En primer lugar hay estudios que destacan la importancia de la formación continua del profesorado universitario como una vía hacia la mejora de la práctica docente. En este sentido, un informe elaborado por Center for the Study of Teaching and Policy (Darling-Hammond, 1999) pone de manifiesto que en el proceso de aprendizaje de los alumnos, intervienen muchos factores relacionados con el perfil profesional del profesorado.

Otros estudios (Guyton \& Farokhi, 1987) han encontrado una fuerte relación consistentemente positiva entre los cursos de formación del profesorado y la eficacia de la práctica docente de éstos. Al mismo tiempo se reporta un efecto positivo constante de la formación del profesorado en las calificaciones de supervisión y el aprendizaje del estudiante (Ferguson \& Womack, 1993, entre otros). Otros estudios ponen de manifiesto que la formación de manera continuada de los docentes es fundamental para la mejora de sus prácticas en el aula. García Llamas (1998), Trigwell, Prosser \& Waterhouse 
(1999) y Ho, Watkins \& Kelly (2001) sugerían que la formación continua podría indicar que no es sólo el conocimiento adquirido con el desarrollo profesional continuo (que puede representar los más recientes avances en la base de conocimientos), sino también la influencia que esto tiene sobre la motivación y el entusiasmo del profesor por el aprendizaje, lo que repercute en el aumento de rendimiento de los estudiantes.

En segundo lugar se analizan las metodologías formativas utilizadas en las actividades de formación del profesorado universitario y sus repercusiones sobre la actividad docente. Un estudio realizado por Gibbs \& Coffey (2004) enumera las metodologías de formación profesional empleadas entre las cuales destacan: el debate, la retroalimentación, seminarios y conferencias sobre la enseñanza y actividades de mentoring en los departamentos.

Si hasta hace no mucho tiempo, los programas de formación universitaria se centraban en enseñar al profesorado metodologías centradas en los aprendizajes del alumnado, en los últimos años, con la incorporación del enfoque por competencias, el currículum formativo ha cambiado. Yáñiz (2008, 11) considera que «el enfoque competencial no pide un cambio drástico en la formación que se viene realizando, sino más bien la intensificación y ampliación de la misma, junto con un enfoque marcadamente reflexivo, abierto y dialogal». Rué (2007) añade que el trabajo por competencias cambia el desarrollo de los nuevos planes de formación y García San Pedro (2009) destaca las consecuencias que el modelo competencial tiene sobre la educación superior.

Siguiendo estos principios, en España, algunas de las universidades incluyen en sus planes estratégicos, propuestas de formación adaptadas a la creación del EEES que vienen a mejorar la formación pedagógica del profesorado universitario. Un ejemplo en este sentido es el programa desarrollado en la Universidad de Deusto (UD) en el proyecto «Formación y Desarrollo del profesorado» que pretende lograr la formación y el desarrollo del profesorado según un Plan de Formación y Desarrollo del Profesorado para la incorporación del Modelo de Formación UD» (Universidad de Deusto, 2001). Según Elexpuru, Martinez, Villardón \& Yáñiz, (2009) el modelo integra las orientaciones y consecuencias previstas en el proceso de creación del EEES.

Otra experiencia, entre muchas otras existentes al nivel español, es la de la Universidad de Zaragoza, que tiene como objetivo un verdadero cambio de modelo educativo caracterizado, especialmente, por estar centrado en el aprendizaje del alumno y orientado hacia la adquisición de competencias (Actividades de formación para el profesorado universitario-ICE, 2009). 
Al nivel de las universidades catalanas, existen instituciones encargadas con la formación del profesorado universitario que pertenecen a las mismas universidades. Por ejemplo la Universidad de Barcelona, la Universidad Rovira i Virgili (URV), la Universidad Politécnica de Catalunya (UPC) tienen un Instituto de Ciencias de la Educación que se encarga de la formación. Las mismas funciones las desempeñan en la Universidad Autónoma de Barcelona, la Unidad IDES y el E-learn Center en el caso de la Universitat Oberta de Catalunya (UOC). El hilo conductor de todos los programas de formación consiste en la diversidad de la oferta en términos de contenido y metodologías de trabajo.

Analizando las ofertas formativas de estos centros, destacamos la preocupación por la mejora de la docencia universitaria a través de proporcionar al docente las herramientas más adecuadas para el desempeño de sus tareas. Se implementan en este sentido dos tipos de formación:

- formación inicial con un contenido básico necesario para iniciar la docencia universitaria;

- formación continuada con herramientas diversas, desde cursos de larga duración (a veces en formato Máster) hasta cursos y talleres puntuales que responden a las necesidades inmediatas del profesorado, ofreciendo cada vez más formación a medida, ligada a facultades en concreto y a proyectos y planes particulares.

En cuanto al contenido de estas ofertas formativas, destacan los aspectos relacionados con la actividad de enseñanza- aprendizaje: confección de guías docentes (UAB), difusión y transferencia de buenas prácticas de enseñanza-aprendizaje (UOC), el coaching en la docencia (URV). Sin embargo se constata una escasa presencia de las ofertas formativas en temas relacionados con la adaptación de la evaluación por competencias a las EEES. Aunque haya algunas referencias a la evaluación en la mayoría de los casos (UB; UAB; UPC) se hace énfasis en la evaluación de los aprendizajes.

El escaso interés frente a la formación del profesorado universitario en temas de evaluación por competencias explica los resultados de los estudios llevados a cabo por Pastor (2004) y Cano, Ion, \& Compañó (2010) donde se revela la falta de información y formación que el profesorado tiene sobre estos aspectos. Si en el primer estudio, un $49,22 \%$ de los encuestados considera que el conocimiento sobre estas cuestiones es deficiente, en el segundo mencionado el profesorado de las universidades catalanas reconoce que no realiza una práctica docente evaluativa basada en competencias por falta de conocimiento suficientemente consolidado sobre los requisitos de ECTS. 
Así pues, garantizar la cualificación y competencia del profesorado representa un aspecto clave del proceso de renovación universitaria. En él tienen una clara responsabilidad las instituciones pero, por lo que se ha señalado, y pese a existir una fuerte oferta formativa, el profesorado no percibe dichas acciones como suficientes y/o eficientes.

\section{MÉTODO}

\subsection{El estudio. El punto de partida}

En este contexto universitario planteamos la investigación: «Evaluación de competencias de los estudiantes universitarios ante el reto del EEES: descripción del escenario actual, análisis de buenas prácticas y propuestas de transferencia a diferentes entornos» (I+D -SEJ2007-65786EDUC). Para aproximarnos al panorama general de las prácticas evaluadoras de las universidades, se ha aplicado un cuestionario a una muestra de profesores universitarios de Cataluña. La muestra proviene de la selección de tres universidades catalanas: Universidad de Barcelona (UB), Universidad Rovira y Virgili (URV) y la Universidad Oberta de Catalunya (UOC) y de cinco titulaciones tal como se indica en la tabla 1.

\begin{tabular}{|c|c|c|c|c|}
\hline & UB & URV & UOC & TOTAL \\
\hline Humanidades & 72 & 15 & 6 & 93 \\
\hline $\begin{array}{c}\text { Ciencias } \\
\text { experimentales }\end{array}$ & 85 & 9 & - & 94 \\
\hline $\begin{array}{c}\text { Ciencias } \\
\text { sociales }\end{array}$ & 75 & 14 & 4 & 93 \\
\hline $\begin{array}{c}\text { Enseñanzas } \\
\text { Tecnológicas }\end{array}$ & - & 16 & - & 114 \\
\hline $\begin{array}{c}\text { Ciencias } \\
\text { de la salud }\end{array}$ & 98 & 70 & 14 & 414 \\
\hline TOTAL & 330 & & & 387 \\
\hline $\begin{array}{c}\text { TOTAL } \\
\text { RESPUESTAS }\end{array}$ & & 16 & & \\
\hline
\end{tabular}

Tabla 1. Distribución de la muestra por universidades y titulaciones. 
Atendiendo a dos variables (universidad y campo de conocimiento) y aceptando un margen de error de 0,07 (con alpha 0,05), se llegó a determinar una muestra de 905 profesores/as. Como dicha muestra resultaba excesiva para la envergadura del grupo de investigación, por motivos de accesibilidad, se decidió limitar la recogida a tres universidades públicas diferentes (la UB, por su gran tamaño, diversidad de estudios y antigüedad y cuyo profesorado en sí mismo puede considerarse representativo del sentir del profesorado universitario catalán; la URV por ser pequeña y hallarse en una demarcación territorial muy diferente a Barcelona y la UOC, por ser abierta y de reciente creación). Los cuestionarios han sido validados mediante prueba piloto. De los 387 cuestionarios entregados, se respondieron 211.

De todos los académicos entrevistados sólo una minoría $(27,60 \%)$ ha reconocido haber realizado una experiencia de evaluación por competencias, declarándose el resto todavía centrados en una evaluación de los aprendizajes. Entre los motivos aducidos para no realizar prácticas de evaluación por competencias destacan:

a) estar esperando algún tipo de consigna o política institucional, con directrices claras sobre qué hacer y cómo (hay que tener presente que el cuestionario se administró antes de que la mayoría de universidades hubiesen empezado oficialmente los estudios de grado) y

b) no sentirse preparado, no tener formación para acometer este tipo de tarea.

Por este motivo nos propusimos una nueva acción en el marco de la investigación: administrar una entrevista escrita, abierta, a directores de los ICEs, con el objetivo de recoger información sobre las políticas y las actividades de formación, que eran los aspectos causantes de la falta de evaluación por competencias. Se analizan las prácticas formativas del profesorado universitario existentes en las universidades catalanas, destacando los temas prioritarios de esta formación, las metodologías empleadas y las dificultades que aparecen en el proceso de formación del profesorado universitario con relación a la evaluación de las competencias.

El análisis aportará más información sobre la formación del profesorado universitario ya que hemos visto que aunque hay varios estudios que se encargan de analizar este aspecto, pocos consiguen analizar esta formación desde la perspectiva de evaluación por competencias. Esto permitirá orientar la toma de decisiones por parte de los líderes académicos sobre el proceso de formación del profesorado universitario y dará pistas a los res- 
ponsables de institutos de formación de las universidades para la planificación de sus acciones formativas.

\subsection{El diseño metodológico}

La metodología empleada es de corte cualitativo y consiste en la aplicación de un guión de entrevista a los responsables de la formación de las universidades catalanas. Las universidades participantes en el estudio son las universidades: Universidad de Barcelona (UB); Universidad Autónoma de Barcelona (UAB); Universitat Oberta de Catalunya (UOC); Universidad Rovira i Virgili Tarragona (URV); Universidad Politécnica de Catalunya (UPC).

Se entrevista a los coordinadores de los Institutos de Ciencias de la Educación (ICE) u organismos con las mismas funciones de las 5 universidades, considerando éstos como los encargados de impulsar y apoyar desde el punto de vista académico la implementación de los nuevos planteamientos derivados del EEES, tal como se observa en la tabla siguiente:

\begin{tabular}{|l|c|l|l|l|l|}
\hline Universidad & UB & UAB & UPC & URV & UOC \\
\hline $\begin{array}{l}\text { Participan- } \\
\text { tes en el es- } \\
\text { tudio }\end{array}$ & $\begin{array}{l}\text { Responsa- } \\
\text { ble ICE }\end{array}$ & $\begin{array}{l}\text { Responsa- } \\
\text { ble IDES }\end{array}$ & $\begin{array}{l}\text { Responsa- } \\
\text { ble ICE }\end{array}$ & $\begin{array}{l}\text { Responsa- } \\
\text { ble ICE }\end{array}$ & $\begin{array}{l}\text { Responsa- } \\
\text { ble E-learn } \\
\text { Center }\end{array}$ \\
\hline
\end{tabular}

Tabla 2. Los informantes clave participantes en la investigación

A cada responsable de ICE se le ha aplicado una entrevista semi-estructurada con preguntas abiertas. Los tópicos y las categorías de análisis de las entrevistas se pueden sistematizar en la tabla 3: 


\begin{tabular}{|c|c|}
\hline Tópicos & Categorías de análisis \\
\hline \multirow{4}{*}{$\begin{array}{l}\text { 1. Formación del profesorado para } \\
\text { el sistema de trabajo con las compe- } \\
\text { tencias }\end{array}$} & $\begin{array}{l}\text { a. La importancia de la formación del pro- } \\
\text { fesorado en temas relacionados con la en- } \\
\text { señanza-aprendizaje por competencias }\end{array}$ \\
\hline & $\begin{array}{l}\text { b. Estrategias formativas empleadas para } \\
\text { promover esta formación }\end{array}$ \\
\hline & $\begin{array}{l}\text { c. La participación del profesorado en estos } \\
\text { cursos }\end{array}$ \\
\hline & $\begin{array}{l}\text { d. Oferta y demanda de formación ( tópicos } \\
\text { más frecuentes) }\end{array}$ \\
\hline \multirow{3}{*}{$\begin{array}{l}\text { 2. Oportunidad y relevancia de la } \\
\text { oferta formativa en competencias }\end{array}$} & $\begin{array}{l}\text { a. Temas que se hayan ofrecido en los pla- } \\
\text { nes de formación. }\end{array}$ \\
\hline & $\begin{array}{l}\text { b. La adecuación de estos cursos a las nece- } \\
\text { sidades del profesorado }\end{array}$ \\
\hline & $\begin{array}{l}\text { c. Aspectos a mejorar en la formación por } \\
\text { competencias del profesorado universitario }\end{array}$ \\
\hline
\end{tabular}

Tabla 3. Tópicos y categorías de análisis de las entrevistas

\section{RESULTADOS}

Las respuestas recibidas de parte de los participantes al estudio se han categorizado y se han analizado en función de los tópicos propuestos a priori.

Vamos a proceder a desgranar algunos de los resultados hallados en cada uno de los aspectos analizados.

\subsection{La formación del profesorado para el trabajo por competencias}

Analizando las respuestas de los responsables de los institutos de formación del profesorado universitario (ICE- IDES) se puede observar que se tienen opiniones definidas sobre el trabajo por competencias. 
Los entrevistados insisten en aclarar que se ha fomentado la formación en cursos para la adaptación de la docencia a los retos de EEES. Si la demanda va orientada hacia la planificación de la docencia por competencias, no se observa el mismo interés en cuanto a la formación para la evaluación de las competencias. De los casos analizados destacamos el caso UAB que habla de un interés explicito hacia este aspecto. En algunos de los otros casos (UPC; UB o URV) se afirma que el profesorado está interesado en formarse en la evaluación en general. Técnicas para potenciar la comunicación y la participación en el aula, o la gestión del tiempo, son otros enfoques solicitados en los cursos de formación.

Un aspecto que llama la atención se refiere a la oferta formativa relacionada con la formación en estrategias de trabajo en equipos profesionales (caso UB por ejemplo), las herramientas tecnológicas para la enseñanza: moodle y sus herramientas (caso UB; URV; UAB), la confección de guías docentes (UAB; UPC; UOC) o las competencias transversales (UOC).

También se insiste sobre el incremento de solicitudes para inscribirse en programas de formación (por ejemplo: programa de Máster UB; ProFi UPC; Curso IDES) y especialmente en cursos obligatorios para la docencia universitaria como es el caso de IDES de la UAB. Se trata de un programa de acreditación que tiene como objetivo desarrollar las competencias básicas del profesorado universitario y que se configura en base a cursos obligatorios y talleres optativos a elegir según la oferta formativa.

Refiriéndose a la importancia que se ofrece a la formación en el contexto universitario actual, los entrevistados coinciden en considerarlo un proceso «prioritario» y en vincular la formación con una docencia de calidad:

«Es indispensable una formación integral, inicial y a lo largo de toda la carrera profesional de los docentes para formar profesionales reflexivos preparados para abrirse camino en un entorno de cambio e innovación permanente» (UAB).

El enfoque por competencias, la necesidad de formar profesionales adaptados a los nuevos contextos laborales, adaptables a la sociedad actual, es otro motivo por el interés hacia una formación adecuada por competencias:

«Es fundamental. En la nueva estructura del EEES, el diseño por competencias es una herramienta básica para garantizar programas formativos de calidad y equiparables a nivel europeo. Además, el rediseño de las asignaturas, ahora por competencias, se convierte en un catalizador para la innovación metodológica» (UOC) 
Un apartado amplio dentro de la entrevista se ha centrado en las modalidades formativas y de evaluación empleadas en estos cursos de formación. Éstas tienen múltiples formatos. Una síntesis de las respuestas de los entrevistados se muestra en la tabla siguiente.

\begin{tabular}{|c|c|c|c|c|}
\hline UAB & UB & UPC & URV & UOC \\
\hline $\begin{array}{l}\text { - Formación } \\
\text { de medida; } \\
\text { - Talleres con } \\
\text { el objetivo de } \\
\text { presentar me- } \\
\text { todologías de } \\
\text { en se ñan za- } \\
\text { aprendizaje; } \\
\text { - "Observa- } \\
\text { ción al aula»- } \\
\text { los profesores } \\
\text { se organizan } \\
\text { en grupos de } \\
\text { tal manera } \\
\text { que cada uno } \\
\text { pueda asistir a } \\
\text { las sesiones de } \\
\text { los demás; } \\
\text { - Jornadas } \\
\text { temáticas- ac- } \\
\text { tividades in- } \\
\text { tensivas de } \\
\text { formación }\end{array}$ & $\begin{array}{l}\text { - Formación } \\
\text { permanente } \\
\text { en dos edicio- } \\
\text { nes al año en } \\
\text { función de la } \\
\text { demanda de } \\
\text { los centros; } \\
\text { - Talleres; } \\
\text { - Actividades } \\
\text { de asesora- } \\
\text { miento. }\end{array}$ & $\begin{array}{l}\text { - Elaboración } \\
\text { de documenta- } \\
\text { ción orientada } \\
\text { a los equipos } \\
\text { directivos en- } \\
\text { cargados de la } \\
\text { elaboración de } \\
\text { las guías do- } \\
\text { centes y a los } \\
\text { profesores } \\
\text { - Diseño de un } \\
\text { plan de forma- } \\
\text { ción de las } \\
\text { competencias } \\
\text { genéricas de la } \\
\text { universidad, } \\
\text { flexible } \\
\text { abierto; } \\
\text { - Visitas a cada } \\
\text { uno de los cen- } \\
\text { tros docentes } \\
\text { para analizar } \\
\text { de forma per- } \\
\text { sonalizada y } \\
\text { conjunta las } \\
\text { necesidades } \\
\text { del profesora- } \\
\text { do para la } \\
\text { adaptación a } \\
\text { las EEES; } \\
\text { - Actividades } \\
\text { personalizadas; }\end{array}$ & $\begin{array}{l}\text { - Seminarios; } \\
\text { - Talleres } \\
\text { - Jornadas Es- } \\
\text { pecíficas para } \\
\text { cada centro }\end{array}$ & $\begin{array}{l}\text { - Seminario } \\
\text { de innovación } \\
\text { docente; } \\
\text { - Talleres prác- } \\
\text { ticos semipre- } \\
\text { senciales en } \\
\text { los que los } \\
\text { profesores tu- } \\
\text { vieran que ela- } \\
\text { borar algo } \\
\text { (una parte del } \\
\text { plan docente, } \\
\text { una actividad, } \\
\text { etc.) } \\
\text { - Cursos vir- } \\
\text { tuales sobre el } \\
\text { diseño de cur- } \\
\text { sos y progra- } \\
\text { mas por com- } \\
\text { petencias }\end{array}$ \\
\hline
\end{tabular}




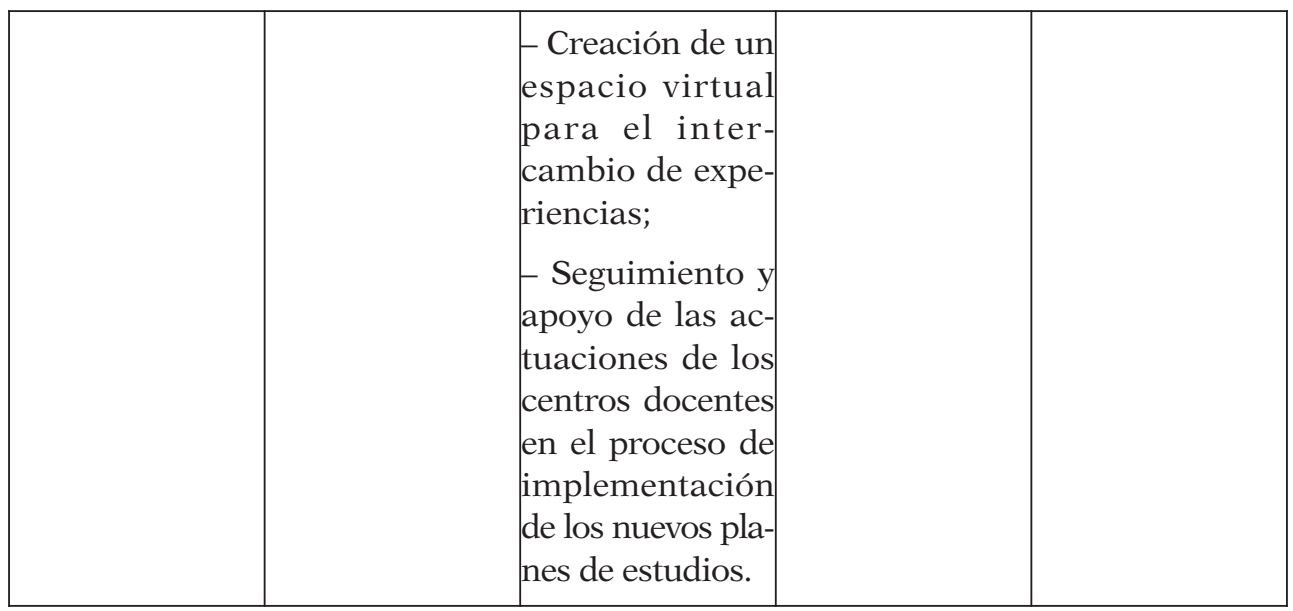

Tabla 4. Estrategias de formación del profesorado en las universidades estudiadas

En general, se observa que desde los centros de formación se desarrollan dos tipos de formación: por un lado una formación estandarizada, prescrita, establecida en momentos determinados (junio, enero o a lo largo del curso académico), con un formato preestablecido (cursos, talleres) y, por otro lado, una formación a medida, más personalizada, a petición de los centros docentes y/o de los grupos de innovación docente (básicamente asesoramientos pero también talleres, seminarios, cursos puntuales).

\subsection{Oportunidad y relevancia de la oferta formativa en competencias}

La participación del profesorado en los cursos de formación sobre las competencias en todas las universidades estudiadas es apreciada por sus promotores, como «muy positiva». Los entrevistados mencionan que en general los profesores se interesan por su formación pero sin embargo...

«Hay algunas excepciones en que el profesorado no sentía la necesidad de formarse en competencias docentes o bien en que la formación se ofrecía en épocas de sobrecarga docente» (UPC).

La falta de tiempo, de motivación o de sensibilización para la formación son, entre otras, poderosas razones para explicar el bajo interés o participación hacia la formación sobre competencias:

A pesar que a veces se ha incentivado desde la dirección de los estudios, es necesario que el profesorado disponga del tiempo suficiente para hacer es- 
tas formaciones y que, además del aprendizaje que les suponga, puedan tener alguna acreditación que dé valor curricular a la misma. Inicialmente muestran interés, pero hay una gran sensibilización para facilitar el cambio. (UOC)

Si nos centramos específicamente en la formación sobre la evaluación por competencias, observamos que los cursos que se piden con prioridad se centran en torno a la evaluación con grupos grandes, por ejemplo sobre cómo organizar los grupos para el trabajo de las competencias específicas o generales. Sin embargo, algunas de las entrevistas muestran que hay más propuestas de formación dirigidas hacia la enseñanza- aprendizaje de las competencias y la evaluación de los contenidos que orientada hacia la evaluación por competencias. También hay casos donde la evaluación de las competencias no forma parte del currículum formativo.

Al preguntar sobre si los nuevos planteamientos requeridos por el EEES han marcado un cambio de paradigma en cuanto a la formación y el interés por el desarrollo profesional de los docentes, las respuestas han variado significativamente.

Por un lado se considera que no se ha percibido ningún tipo de cambio (el caso de la UOC). Por otro lado se afirma que sobre todo lo que ha cambiado es la mirada de los profesores sobre su propia práctica docente. La opinión de uno de los entrevistados resume este punto de vista:

"Las necesidades formativas del profesorado han cambiado con el planteamiento del EEES que ha supuesto un cambio de mirada, poniendo el énfasis en la tarea del alumno, en su aprendizaje y en sus competencias. El ECTS ha hecho que las asignaturas valoren y asignen una dedicación de trabajo que cada alumno deberá realizar para alcanzar los objetivos establecidos en la materia. Este cambio de perspectiva del proceso de enseñanza - aprendizaje hace que la formación del profesorado universitario, necesariamente, cambie de perspectiva» (UPC).

\subsection{Propuestas de mejora en la formación por competencias}

Un apartado importante de la entrevista intenta averiguar si la experiencia formativa del profesorado necesita mejorar después de las primeras experiencias de adaptación al EEES.

En este sentido destacan opiniones que se refieren no solo al contenido ofrecido a través de la oferta formativa sino también a los formatos y las metodologías empleadas que tengan como resultado un cambio en la cultura docente del profesorado universitario. 
Algunas propuestas van resumidas en la tabla siguiente:

\begin{tabular}{|c|c|c|c|c|}
\hline UAB & UB & UPC & URV & UOC \\
\hline $\begin{array}{l}\text { - Apostar por } \\
\text { modalidades } \\
\text { que acaben } \\
\text { con un proyec- } \\
\text { to, para facili- } \\
\text { tar la transfe- } \\
\text { rencia }\end{array}$ & $\begin{array}{l}\text { - Diversificar } \\
\text { modalidades; } \\
\text { - Apostar por } \\
\text { proyectos }\end{array}$ & $\begin{array}{l}\text { - F o r m a to s } \\
\text { semi-presen- } \\
\text { ciales de for- } \\
\text { mación; } \\
\text { - Presentación } \\
\text { de casos que } \\
\text { no han resul- } \\
\text { tado exitosos y } \\
\text { que no se de- } \\
\text { ben repetir y } \\
\text { de casos de } \\
\text { buenas prácti- } \\
\text { cas }\end{array}$ & $\begin{array}{l}\text { - Comple- } \\
\text { mentar la for- } \\
\text { mación con } \\
\text { medidas insti- } \\
\text { tucionales de } \\
\text { apoyo a la im- } \\
\text { plementación } \\
\text { de cambios en } \\
\text { el aula; } \\
\text { - Ofrecer más } \\
\text { casos reales y } \\
\text { ejemplos de } \\
\text { buenas prácti- } \\
\text { cas; }\end{array}$ & $\begin{array}{l}\text {-Hacer un se- } \\
\text { guimiento de } \\
\text { la formación, } \\
\text { con trabajo } \\
\text { por parte de } \\
\text { redes }\end{array}$ \\
\hline
\end{tabular}

Tabla 5. Propuestas de mejora de los programas de formación para la evaluación por competencias

Podemos observar que las propuestas de mejora van orientadas hacia un cambio de paradigma evaluativo, que sea capaz de realizar una transformación real en la práctica del profesorado, acentuando las buenas prácticas y propiciando la innovación en el marco de las titulaciones. Se desea apostar en el futuro por experiencias compartidas entre los profesionales y por la formación en formatos semi-presenciales.

\section{DISCUSIÓN Y CONCLUSIONES}

Según los participantes, el profesorado se muestra abierto a ponerse al día con las nuevas metodologías de enseñanza-aprendizaje y evaluación centradas en competencias del estudiante. Nuestros resultados se inscriben en la misma línea de otros estudios que demuestran las ventajas de los programas de desarrollo profesional para la práctica del profesorado (Corcoran, Shields \& Zucker, 1998; Garet, Birman, Porter, Desimore, Herman \& Yoon, 1999, National Staff Development Council, 1995; Yáñiz, 2008; entre otros).

Conviven las acciones formativas más reflexivas con las más instrumentales pero, probablemente, estas segundas ganan en presencia a las pri- 
meras. Ello nos lleva a alertar del riesgo de formaciones excesivamente instrumentales, sin establecer las bases teóricas de metodologías y recursos, sin dotarlas de un componente contextual ni reflexivo. Barnett (2001) se muestra especialmente sensible a este punto y nos advierte de los riesgos de centrarnos en la dimensión técnica de los discursos por competencias frente a la riqueza de la enseñanza universitaria, que ha de incorporar la duda, la comprensión, la reflexión y no únicamente mecanismos de aplicación eficiente de procesos prescritos por otros. De ahí la importancia de las estrategias formativas que se articulen en las diversas modalidades.

Por otro lado hemos visto que la formación del profesorado universitario abarca una variedad de modalidades, todas construidas para favorecer el modelo del profesorado que el EEES requiere. En la mayoría de las universidades, todavía hay un abanico bastante variado de prácticas formativas «tradicionales» (cursos en formatos presenciales, de larga duración, planes de formación extensos) y pocas actividades de acompañamiento, de trabajo cooperativo o de intercambio de experiencias. De hecho, autores como Elmore \& Burney, (1999); Hightower, (2002); Young \& Kim, (2010), destacan las potencialidades del coaching como una de las estrategias para la mejora del uso de la evaluación centrada en competencias. Las mismas potencialidades se les asignan a las prácticas de aprendizaje colaborativo y comunitario. Estrategias que impliquen la colaboración entre varios profesionales, el intercambio de experiencias y el acompañamiento entre iguales empiezan a abrirse camino entre las metodologías formativas en las universidades, aunque será necesario que sigamos avanzando en ellas. Autores como McLaughin \& Talbert, (2001) ponen de manifiesto que la construcción de comunidades de aprendizajes representa una de las estrategias para reformar las prácticas docentes. Mientras tanto, el uso de las estrategias colaborativas basadas en observación entre iguales de las prácticas de evaluación tienen repercusiones positivas sobre la actividad en el aula, idea reforzada por autores como Cizek, Fitzagerald, \& Rachor (1995/96) y McMillian (2002). Por lo tanto, ofrecer experiencias formativas orientadas hacia la reflexión, grupales y comunitarias, representa una buena estrategia para el cambio que se requiere por los ECTS.

Los programas de formación del profesorado universitario, reflejan las necesidades de evaluación del profesorado en sus clases (Stiggins, 1991 e Impara, Plake, \& Fager, 1993) y por otro lado la participación a los cursos organizados es discontinua (Herman \& Dorr-Bremme, 1983). Las prácticas docentes del profesorado universitario se ven influenciadas muchas veces por factores organizativos e implican un cierto aislamiento y privacidad dominados tradicionalmente por normas culturales (Little, 1990) lo que hace que las prácticas de evaluación centradas en competencias tiendan a ser individuales y poco compartidas. 
En este sentido, nuestros entrevistados consideran que se intenta dar progresivamente mayor peso a la formación a demanda, a medida, especialmente si viene de equipos docentes. Estas propuestas, ligadas a cursos o proyectos concretos, son más fácilmente transferibles. Pese a ello, incluso en las modalidades de grupos de trabajo, tal y como recogen Moreno, Quesada \& Pineda (2010), existen dificultades para aplicar los aprendizajes a las aulas. Por lo tanto, velar por la transferencia es una de las asignaturas pendientes.

Sin embargo, la formación profesional del profesorado, tal como hemos visto, se ve dificultada por varios factores, entre los cuales se remarca la falta de tiempo o de motivación del profesorado participante. Los entrevistados de nuestro estudio consideran que para mejorar las prácticas evaluadoras del profesorado hacia el uso de técnicas no-tradicionales de evaluación supone un consumo de tiempo mayor que el uso de las técnicas clásicas de tipo papel-lápiz (Borko, Mayfield, Marion, Flexer \& Cumbo, 1997; Hall \& Hewitt-Gervais, 2000; Cano, Ion \& Compaño, 2010). Ésta es la razón por la cual plantear actividades formativas en periodos de menos carga docente $o$ en periodos no-lectivos o adoptar medidas de acompañamiento en momentos de máxima necesidad puede ser una buena estrategia para una oferta formativa flexible.

Por último, consideramos que pese a que se dedican grandes esfuerzos a formación, faltan todavía estudios consistentes, sobre la transferencia e impacto de la misma (Weimer \& Lenze, 1997; Gilbert \& Gibbs, 1999; Gibbs \& Coffey, 2004), tal y como hemos indicado anteriormente.

En conclusión, de las respuestas obtenidas por los informantes clave en varias universidades catalanas podemos intuir que, aunque se observa que las universidades tienen como preocupación cada vez más presente la formación del profesorado, aún predominan las estrategias formativas tradicionales y los temas «clásicos». Todavía se necesita más esfuerzo para ofrecer una formación coherente y adecuada a los nuevos retos del EEES. 


\section{REFERENCIAS BIBLIOGRÁFICAS}

Alonso, M. I. (2010). Elaboración de una guía con casos de aplicación práctica para el desarrollo- evaluación de competencias en los estudiantes universitarios e indicadores para el estudio de la calidad del proceso. Madrid: Dirección General de Universidades de la Secretaría de Estado de Universidades e Investigación; Programa de Estudios y Análisis.

Barnett, R. (2001). Los límites de la competencia: el conocimiento, la educación superior y la universidad. Madrid: Gedisa.

Borko, H.; Mayfield, V.; Marion, S.; Flexer, R. \& Cumbo, K. (1997). Teachers'developing ideas and practices about mathematics performance assessment: Successes, stumbling blocks, and implications for professional development. CSE Technical Report 432. Los Angeles: NCRESST.

Cano, E. (2008). La evaluación por competencias en la educación superior. Profesorado. Revista de curriculum y formación del profesorado. Disponible en: http://www.ugr.es/local/recfpro/rev123CO L1.pdf [consulta: 2010, 17 de octubre].

Cano, E., Ion, G. \& Compañó, P. (2010). Assessment practices at Catalonian Universities: from learning to competencies approach. Paper presentado a European Conference on Educational Research, 24-28 de Agosto de 2010, Helsinki.

Cano, E. (Coord.) (2011). Buenas prácticas en la evaluación de competencias. Cinco casos en educación superior. Barcelona: Laertes.

Cano, E.; Lleixà, T.; Ion, G. y Tierno, J.M. (2011). Criterios para una buena práctica de evaluación, en Pagés, T. (Co- ord.) Buenas prácticas docentes en la universidad. Modelos y experiencias en la Universidad de Barcelona. Barcelona: ICE/Octaedro, 86-97.

Cizek, G, Fitzagerald, S. \& Rachor, R. (1995/96). Teachers'assessment practices: preparation, isolation and the kitchen sink. Educational Assessment, 3 (2), 159-179.

Corcoran, T.; Shields, P. \& Zucker, A., (1998). SSIs and professional development for teachers. Menlo Park, CA: SRI International.

Darling-Hammond, L. (1999). Teacher Quality and Student Achievement: A Review of State Policy Evidence. Washington: Center for the Study of Teaching and Policy University of Washington.

De Miguel, M. (coord.) (2004). Modalidades de enseñanza centradas en el desarrollo de competencias. Oviedo: Ediciones de la Universidad de Oviedo.

Edwards, M.; Donderis, V. \& Ballester, E. (2005). La participación del profesorado y de los estudiantes: factor clave para el éxito del proceso de convergencia. Ponencia presentada en: XIII Congreso Universitario de Innovación Educativa en Enseñanzas Técnicas. 21, 22 y 23 de septiembre, Maspalomas (Gran Canaria).

Elexpuru, I.; Martinez, A.; Villardón L.; Yáñiz, C. (2009). Descripción y evaluación del plan de formación y desarrollo del profesorado de la Universidad de Deusto. Red U. Revista de Docencia Universitaria, 3 [en línea]. Disponible en: http://www.um.es/ead/Red_U/3/ [consulta: 2010, 23 agosto].

Elmore, R. \& Burney, D. (1999). Investing in teacher learning. Staff development and instructional improvement, in Dar- 
ling- Hammond, L. \& Sykes, G. (eds.) Teaching as the learning profession. San Francisco: Jossey-Bass, 263-291.

Espinosa J. K.; Jiménez, J.; Olabe, Y. \& Basogain, X. (2010). Innovación Docente para el Desarrollo de Competencias en el EEES [en línea]. Disponible en: http:/campus.usal.es/ ofeees/ARTICULOS/p216.pdf [consulta: 2010, 15 de octubre].

EUA (2005). Trends IV: European Universities Implementing Bologna. Bruselas: EUA [en línea]. Disponible en: http://www.eua.be/eua/jsp/en/upload/TrendsIV_FINAL.1117012084971.p df [Consulta: 2010, 12 de junio].

EUA (2010). Trends V: Universities shaping the European Higher Education Area. Bruselas: EUA [en línea]. Disponible en: http://www.eua.be/fileadmin/user_upload/files/Publications/Final_Trends_Report__May_10.pdf [Consulta: 2010, 12 de junio].

Ferguson, P. \& Womack, S .T. (1993). The impact of subject matter and education coursework on teaching performance. Journal of Teacher Education, 44 (1), 55-63.

Gairín, J. et al. (2009). Guía para la evaluación de competencias en el área de ciencias sociales. Barcelona [en línea]. Disponible en: www.aqu.cat. [Consulta: 2011, 10 de marzo].

García Llamas, J. L. (1998). La Formación Permanente del Profesorado: Motivaciones, Realizaciones y Necesidades. Educación XX1, 1, 129-158.

García-San Pedro, M. J. (2009). El concepto de competencias y su adopción en el contexto universitario. Revista alternativas. Cuadernos de trabajo social. 16, 11-28.

Garet, M. S.; Birman, B.F.; Porter, A.C.; Desimore, L.; Herman, R. \& Yoon,
K.S. (1999). Designing effective professional development: Lessons from the Eisenhower Program. Washington: US Department of Education.

Garmendia, C. (2009). De la construcción del Espacio Europeo de Educación Superior, "Bolonia» y otros demonios. La Cuestión Universitaria, 5, 4-9.

Gibbs, G. \& Coffey, M. (2004). The Impact of Training of University Teachers on their Teaching Skills, their Approach to Teaching and the Approach to Learning of their Students. Active Learning in Higher Education, 5, 87100.

Gilbert, A. \& Gibbs, G. (1999). A proposal for an International Collaborative Research Programme to Identify the Impact of Initial Training on University Teachers. Research and development in higher education, 21, 131-143.

Guyton, E. \& Farokhi, E. (1987). Relationships among academic performance, basic skills, subject matter knowledge and teaching skills of teacher education graduates. Journal of Teacher Education, 38 (5), 37-42.

Hall, K. \& Hewitt-Gervais, C.M. (2000). The application of student portfolios in primary / Intermediate and selfcontained /multi-age team classroom environments: Implications for instruction, learning and assessment. Applied measurement in Education, 13 (2), 209-228.

Herman, J. \& Dorr-Bremme, D. (1983) Uses of testing in the schools: a national profile, in Hathaway, W.E. (ed.) Testing in the schools: New directions for testing and measurement. San Francisco: Jossey-Bass, 7-17.

Hightower, A.M. (2002). San Diego's big boom: District bureaucracy support cul- 
ture of learning. National Center for Research on Evaluation, Standards and Policy. University of Washington.

Ho, A.; Watkins, D.; Kelly, M. (2001). The conceptual change approach to improving teaching and learning: An evaluation of a Hong Kong staff development programme. Higher Education, 42: 143-169.

Impara, J.C.; Plake, B.S. \& Fager, J.J. (1993). Teachers'assessment background and attitudes towards testing. Theory into Practice, 32(2), 113-117.

Javaloyes, J. (2009). La respuesta universitaria a la demanda de formación permanente. Madrid: Dirección General de Universidades del Ministerio de Educación y Ciencia.

Little, J. W. (1990). The persistence of privacy: Autonomy and initiative in teachers'professional relations. Teachers College Record, 91(4), 509-536.

Mateo, J. (2000). La evaluación educativa, su práctica y otras metáforas. Barcelona: ICE- Universidad de Barcelona.

Mclaughin, M. W \& Talbert, J.E. (2001). Professional communities and the work of high school teaching. Chicago: University of Chicago Press.

McMillian, J. (2002). Understanding and improving teachers' classroom assessment decisión making: Implications for theory and practice. Educational Mesurement, 21 (1), 34-43.

Ministros Europeos de Educación (1999). Declaración de Bolonia. [en línea]. http://www.educacion.es/dctm/boloniaeees/documentos/02que/declaracionbolonia.pdf?documentId=0901e72b800 4aa6a [Consulta: 2010, 20 de noviembre].

Moreno, M.V.; Quesada, C.; Pineda, P. (2010). El «grupo de trabajo» como método innovador de formación del profesorado para potenciar la transferencia del aprendizaje. Revista Española de Pedagogía, 246, 281-296.

National Staff Development Council (1995). National Staff development council's standards for staff development. Oxford: $\mathrm{OH}$ Author.

Olmos-Migueláñez, S. \& Rodríguez, M. J. (2006). Diseño del proceso de evaluación de los estudiantes universitarios españoles: ¿responde a una evaluación por competencias en el Espacio Europeo de Educación Superior? Revista Iberoamericana de Educación, 53 (1), 1-13.

Pastor, A. (2004). La viabilidad de las propuestas metodológicas para la aplicación del crédito europeo por parte del profesorado de las universidades españolas, vinculadas a la utilización de las TICs en la docencia y la investigación. Madrid: MEC.

Perrenoud, P. (2004). Diez nuevas competencias para enseñar. Barcelona: Graó.

Rama, C. (2006). El complejo futuro de la evaluación universitaria. Educación XX1 (9), 135-148.

Rué, J. (2007). El Disseny de les titulacions per competències. Ponencia presentada en el Congreso Internacional de Docencia Universitaria, Barcelona, 27 d'abril de 2007.

San Pedro, M. J. (2009). El concepto de competencias y su adopción en el contexto universitario. Revista alternativas. Cuadernos de trabajo social (16), 11-28.

Schön, D.A. (1987). Educating the reflective practitioner: toward a new design in teaching and learning in the professions. San Francisco: Jossey- Bass. 
Stiggins, R. (1991). Relevant classroom assessment training for teachers. Educational Measurement: issues and practice, 10 (1), 7-12.

Suárez, B. (s/f). La formación en competencias: un desafío para la educación superior del futuro [en línea]. Disponible en: http://www.uco.es/organizacion/eees/documentos/normasdocumentos/otros/La\%20formacion\%20en $\% 20$ competencias\%20MEC.pdf [Consulta: 2011, 12 de marzo].

Tejada, J. (2005). El trabajo por competencias en el prácticum: cómo organizarlo y cómo evaluarlo. Conferencia magistral presentada en el VII Symposium Internacional sobre el Practicum y las Prácticas en Empresas en la formación Universitaria, Poio 2005 [en línea]. Disponible en: http://redie.uabc. $\mathrm{mx} / \mathrm{vol} 7 \mathrm{no} 2 / \mathrm{contenidotejada.} \mathrm{[Consulta}$ 2011, 12 de marzo].

Trigwell, K.; Prosser, M. \& Waterhouse, F. (1999). Relations between teachers. Approaches to teaching and students' approaches to learning. Higher Education, 37, 57-70.

Valcárcel, M. (Coord.) (2004). Diseño y validación de actividades de formación para profesores y gestores en el proceso de armonización europea en educación superior. Madrid: MEC.

Villardón, L. (2006). Evaluación del aprendizaje para promover el desarrollo de competencias. Educación siglo $X X I, 24,57-76$.

Weimer, M. \& Lenze, L.F. (1997) Instructional Interventions: A review of the Literature on Efforts to Improve Instruction, in Perry, R.P. \& Smart, J.C. (eds) Effective Teaching in Higher Education: Research and Practice. New York: Agathon Press, 205-240.

Yáñiz, C. (2008). Las competencias en el currículo universitario: implicaciones para diseñar el aprendizaje y para la formación del profesorado. Red U. Revista de Docencia Universitaria [en línea]. Disponible en: http://www.um. es/ead/Red_U/m1/yaniz.pdf [consulta: 2010, 20 de octubre].

Young, V. M. \& Kim, D.H. (2010). Using Assessments for Instructional Improvement. A literature Review. Education Policy 18 (19), 22-47. 


\section{PALABRAS CLAVE}

Evaluación, competencias, educación superior, formación del profesorado, profesorado.

\section{KEY WORDS}

Assessment, competences, higher education, teachers' training, teachers.

\section{PERFIL ACADÉMICO Y PROFESIONAL DE LAS AUTORAS}

Georgeta Ion, profesora lectora del departamento de Pedagogía aplicada de la Universidad Autónoma de Barcelona. Licenciada en Pedagogía por la Universidad de Bucarest y Doctora en Pedagogía por la Universidad de Barcelona. Trabaja en temas relacionados con la Educación Superior, la cultura organizativa y la evaluación. Es miembro del equipo de investigación CAU-AIDA.

Elena Cano, profesora titular del departamento de Didáctica y Organización Educativa de la Universidad de Barcelona. Doctora en Pedagogía por la Universidad de Barcelona. Trabaja en temas relacionados con evaluación y organización educativa. Ha coordinado el proyecto de investigación que ha dado lugar al proyecto del que emerge este trabajo.

Dirección de las autoras: Georgeta Ion

Universidad Autónoma de Barcelona

Bellaterra, G6- 248

E-mail: georgeta.ion@uab.cat

Elena Cano

Universidad de Barcelona

Pg. de la Vall d'Hebron 171, edifici Llevant

08035 Barcelona

E-mail: ecano@ub.edu

Fecha Recepción del Artículo: 08. Diciembre. 2010

Fecha Revisión del Artículo: 20. Abril. 2011

Fecha Aceptación del Artículo: 22. Junio. 2011

Fecha de Revisión para publicación: 22. Noviembre. 2011 